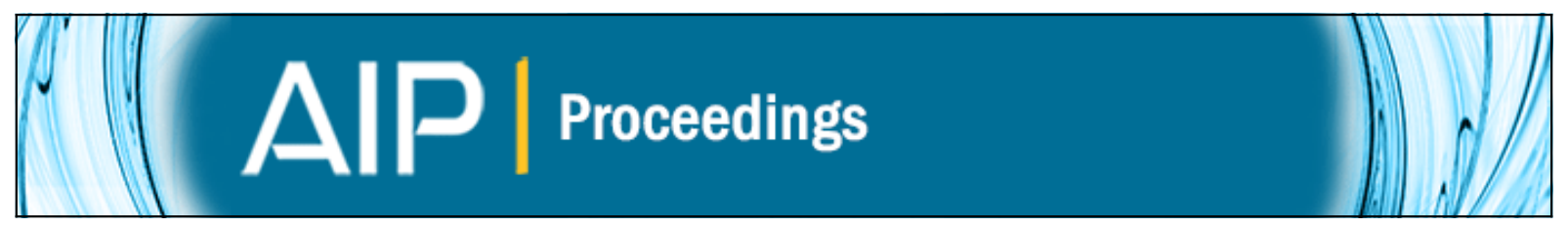

Summary report of Working Group 8: Laser technology for laser-plasma accelerators

Erhard Gaul and Csaba Tóth

Citation: AIP Conference Proceedings 1507, 246 (2012); doi: 10.1063/1.4773701

View online: http://dx.doi.org/10.1063/1.4773701

View Table of Contents: http://scitation.aip.org/content/aip/proceeding/aipcp/1507?ver=pdfcov

Published by the AIP Publishing

Articles you may be interested in

Summary Report of Working Group 1: Laser-Plasma Acceleration

AIP Conf. Proc. 1299, 79 (2010); 10.1063/1.3520427

Summary Report of Working Group 6: Laser-Plasma Acceleration

AIP Conf. Proc. 877, 175 (2006); 10.1063/1.2409132

Summary Report of Working Group: Laser-Plasma Acceleration

AIP Conf. Proc. 737, 223 (2004); 10.1063/1.1842547

Summary Report of Working Group 6: Laser-Plasma Acceleration

AIP Conf. Proc. 647, 165 (2002); 10.1063/1.1524869

Summary report of Working Group 2 on laser-plasma acceleration concepts

AIP Conf. Proc. 569, 35 (2001); 10.1063/1.1384332 


\title{
Summary Report of Working Group 8: Laser Technology for Laser-Plasma Accelerators
}

\author{
Erhard Gaul ${ }^{\mathrm{a}}$ and Csaba Tóth ${ }^{\mathrm{b}}$ \\ ${ }^{a}$ The University of Texas at Austin, 2511 Speedway, Center for High Energy Density Science, Austin, Texas, 78712 , \\ USA \\ ${ }^{b}$ Lawrence Berkeley National Laboratory, Berkeley, CA 94720, USA
}

\begin{abstract}
Laser Technology has long been the limiting and the enabling step for laser plasma accelerators. The work presented here addressed the current and near future laser technology relevant to particle acceleration as well as laser technology challenges for future accelerator facilities. Many laser facilities are operating or will be operating shortly at high intensity, high peak power, and with good beam parameters.
\end{abstract}

Keywords: Laser, Laser particle acceleration, Laser wakefield, High power laser, High intensity laser

PACS: 42.55.-f 42.62.-b 52.35.-g, 52.38.-r, 52.38.Kd

\section{INTRODUCTION}

Most advanced accelerator concepts require electric fields strong enough to separate ions and electrons which under the right conditions drive a plasma wave or to accelerate ions or electrons. The fields are typically created in high peak power laser pulses that are focused to high intensity.

Future laser requirements for laser driven accelerators were established by two recent workshops organized by the Joint Task Force of the International Committee for Future Accelerators (ICFA) and the International Committee on Ultra-High Intensity Lasers (ICUIL), summarized in reference [1]. The challenges from a laser technology point of view are, first, the hard requirements driven by the luminosity of accelerator experiments and second, the wide variety of the pulse energies in combination with a range of repetition rates. The pulse energy for a dielectric laser accelerator might only be milliJoule at $\mathrm{GHz}$ repetition rate, while current ion acceleration concepts strive for kJ pulses at lower shot rate. The core requirements of pulse energy, pulse duration, wavelength, average power and wall plug efficiency, which dominate the choice of laser architecture, material, and technology, vary greatly for different accelerator concepts. It will therefore take several iterations between successful acceleration experiments and technological leaps in laser development to narrow down the laser technology for a future large scale facility.

The laser architecture used for most systems is chirped pulse amplification (CPA), where a short laser pulse is stretched in time by several orders of magnitude, then amplified and then recompressed with reflective optics. This method reduces the intensity during the energy extraction from the amplifier material to a safe level and enables much more compact and efficient high peak power laser systems than without CPA. This 25 years old technique has been used especially in Nd:glass, Ti:sapphire, and other solid state lasers, but is now just as relevant for gas lasers and fiber lasers. More recently, optical parametric chirped pulse amplification (OPCPA) has gained a lot of attention since the parametric amplification process has more flexibility in gain wavelength and bandwidth than suitable laser host materials. In the parametric process the energy from the pump laser beam is transferred to a CPA laser beam in gain crystals. Since this process adds no significant heat source to the crystal, OPCPA could have significant advantages for lasers going to higher repetition rate and average power. Fiber lasers on the other hand have already very high average power along with some of the highest efficiencies. However, they are typically very low in pulse energy $(<\mathrm{mJ})$ and would require beam combining of a significant number of beams to achieve high intensity pulses. $\mathrm{CO}_{2}$ gas lasers can achieve high efficiency and moderated pulse energy and higher repetition rate, but lack the bandwidth for ultra-short pulses and lack the focusability to peak intensities since the wavelength is ten times higher than that of most solid state lasers. 


\section{CHARGE OF THE WORKING GROUP}

This newly created Working Group, WG8, harkens back to AAC 1996, which featured a very successful Working Group entitled "Laser Sources for Particle Acceleration" at a time when multi-terawatt chirped-pulse amplified solid-state laser systems were beginning to heavily influence advanced accelerator research. Today multi-petawatt laser systems are sparking another renaissance in laser-plasma electron and ion acceleration and associated x-ray source development. This Working Group brought together researchers from academia and national laboratories and representatives of commercial vendors of high average- and peak-power laser systems to explore needs and possibilities for the next generation of laser-plasma acceleration research. The goal of the WG was to establish a baseline of what laser systems are available now or in the near future (0-4 years), and identify the trends and requirements in laser development towards higher peak power, higher average power and higher efficiency together with other parameters (pulse duration, wavelength, beam quality, etc...).

\section{CONTRIBUTIONS OF THE WORKING GROUP AND JOINT SESSIONS}

In a plenary talk by Jay Dawson on "High Average Power Lasers for Future Particle Accelerators" it was pointed out that average power combined with high efficiency will be one of the future key requirements. Current high peak power systems show significant improvements but are still several orders of magnitude from the desired specifications. Fiber lasers are technologically near megawatt average power and near the required electrical wall plug efficiency, but do not produce energetic, high peak intensity pulses.

Titanium:sapphire (Ti:Sa) Chirped Pulse Amplification (CPA) lasers are one of the work horses of short pulse high peak power laser system, because it is the currently easiest way to reach relatively high peak power with small, affordable systems. Several Ti:Sa facilities were presented. C. Simon-Boisson and W. Leemans presented the BELLA lasers facility ( $>1 \mathrm{PW}, 40 \mathrm{~J}, 1 \mathrm{~Hz})$ from the construction to the ongoing commissioning at LBNL. F. Canova discussed a similar ongoing upgrade of the DRACO laser at HZDR from $150 \mathrm{TW}$ to $>1 \mathrm{PW}(30 \mathrm{~J}, 1 \mathrm{~Hz})$. E. Chowdhury explained their progress of the SCARLET laser $(0.5 \mathrm{PW}, 1 \mathrm{shot} / \mathrm{min})$ at the Ohio State University with emphasis on high intensity focus. D. Umstadter showed the current status of the Diocles (1 PW, $0.1 \mathrm{~Hz})$ laser at The University of Nebraska and current results. S. Karsch explained - next to a series of current and proposed experiments - the proposed upgrades of the Atlas laser (0.3 PW, few Hz) at LMU Munich by one order of magnitude in peak power at similar repetition rate. K. Krushelnick presented the Hercules laser (University of Michigan) and current experiments. V. Chvykov presented how the transverse lasing in the Ti:Sa affects the scaling of final power amplifier and pointed out how this would affect a proposed upgrade of the Hercules laser $(0.3 \mathrm{PW}, 1 \mathrm{shot} / \mathrm{min})$ to 5 PW. A. Specka (Ecole Polytechnique, France) illustrated how the CILEX facility plans to push Ti:Sa CPA laser to $10 \mathrm{PW}$, which is currently the most ambitious Ti:Sa laser project.

Lasers beside Ti:Sa systems are harder to group. They each have unique properties that are attractive for laser particle acceleration or laser development. The main categories are energetic lasers, fiber lasers, $\mathrm{CO}_{2}$ lasers and OPCPA lasers. The energetic lasers are typically Nd:glass lasers. M. Roth talked about high efficiency side pumped laser and the requirement for energetic pulses for ion acceleration. E. Gaul presented a liquid cooled disk amplifier system that would enable $\mathrm{kJ} \mathrm{Nd}$ :glass lasers at $\sim \mathrm{Hz}$ class repetition rate, which can be either used for pumping Ti:Sa and OPCPA systems or in direct CPA amplification. K. Zeil presented plans at HZDR to construct a diode pumped reprated $(>\mathrm{Hz})$ multi-petawatt $\mathrm{CaF}$ or Yb:glass system for proton therapy. M. Martinez presented the Hybrid OPCPA/mixed glass Texas Petawatt Laser $(180 \mathrm{~J} / 150 \mathrm{fs}$, low shot rate), which is a fully operating facility at The University of Texas. This facility was also available for a tour during a special working group session and for further tours during the workshop.

$\mathrm{CO}_{2}$ lasers operate near $10 \mu \mathrm{m}$ wavelength and typically have bandwidth for ps pulses and operate currently at higher average power than most pulsed solid state lasers. M. Polyanskiy presented proposed $\mathrm{CO}_{2}$ laser development at Brookhaven National Laboratory (BNL) from the current 3-6 ps, TW pulses to $50 \mathrm{TW}$ pulses, by adding OPA seeding as well as self-compression to reduce the pulse duration and by adding optical pumping.

There were several presentations on fiber technology. The plenary talk by J. Dawson addressed many advantages and disadvantages of fibers in regards to accelerators and stated the current technical limits. A. Galvanauskas showed a concept of combining multiple fibers to overcome the energy limitation out of a single fiber. L. Corner on the other hand, presented resonantly enhanced laser wakefield acceleration driven by multiple pulses to limit perhaps the energy requirement of each single drive pulse from a fiber. 
In addition to the most important parameters, such as the peak power, average power, focused intensity and energy, it is also important to have enough finesse in the system. One of the critical laser target interaction, especially with solid target interaction for ion acceleration of injection systems, is the pre-pulse contrast. At an intensity of $10^{21} \mathrm{~W} / \mathrm{cm}^{2}$, which is currently exceeded in multiple facilities, a contrast of ten to twelve orders of magnitude is required to interact with a pristine target. While the contrast was addressed in multiple presentations, $\mathrm{F}$. Canova discussed several techniques to minimize the contrast and measure contrast up to 14 orders of magnitude. $\mathrm{K}$ Flippo presented not only a method of contrast suppression but also how to control the contrast, as it might be important to disturb a solid target with some pre-pulse plasma.

Critical for lasers will also be the time synchronization of laser pulses to existing facilities. M. Babzien presented the challenges of the precision timing between the $\mathrm{CO}_{2}$ laser and the existing linac or $\mathrm{RF}$ injection of the linac at the Accelerator Test Facility at BNL. J. Osterhoff presented timing challenges for timing at the Relativistic Electron Gun for Atomic Exploration at DESY in Hamburg. At both facilities the requirements are to time the laser pulses to a fraction of the pulse duration ( $\sim 10 \mathrm{~s}$ of fs) or perhaps much more precisely.

An overview of matching plasma parameters with laser parameters - based on scaling laws - for a variety of laser-plasma accelerators was presented by C. Schroeder.

\section{LASER TECHNOLOGY DISCUSSIONS}

\section{Titanium:Sapphire Facilities}

Titanium:sapphire is a solid-state laser crystal with the advantage of very broad spectrum which enables for example a $>100$ TW peak power laser with only a few Joules of energy that can fit comfortably on optical tables. A suitable amplifier pump source for the $3.2 \mu$ s lifetime material is a frequency doubled Nd:YAG laser or Nd:glass laser with tens of ns pulse duration. Titanium:sapphire oscillators are commercially available with few fs pulse duration and very broad spectra. Stretchers and compressors have typically gold grating at sufficient size although the 10 PW CILEX system will certainly push the current limit with meter scale compressor gratings.

Crystals have been grown up to $20 \mathrm{~cm}$ in diameter and will likely increase somewhat more in size. The limiting factor that has been discussed in several contributions is the transverse lasing in the amplifier crystal, where amplified spontaneous emission (ASE) starts to extract the stored energy before the seed pulse arrives. The closely related parasitic oscillations can be minimized by surrounding the crystal with absorbing index matching fluid to reduce any backscattering and feedback transverse in the crystal. Side reflection coefficients are typically $0.01-0.1 \%$ which limits the transverse gain to a few 1000. It is also possible to reduce the transverse gain by timing the pump pulse with the laser pulse. It has been suggested that with extraction during pumping (EDP, see Chvykov's paper in this Proceedings) it might be possible to extract kJ energies out of the current size crystals.

Currently Ti:Sa lasers (such as the BELLA laser at LBNL) reach $>1 \mathrm{PW}$ peak power with $1 \mathrm{~Hz}$ repetition rate and $40 \mathrm{~W}$ average power from a commercial supplier. In progress is a similar system upgrade to the DRACO laser at the Helmholtz Zentrum Dresden Rossendorf (HZDR), Germany. There are a number of facilities that have slightly less peak power or operate at lower repetition rate. $100 \mathrm{TW}$ Ti:Sa lasers operate typically at $10 \mathrm{~Hz}$ and $\sim \mathrm{TW}$ Ti:Sa laser operate above $1 \mathrm{kHz}$. Several future Ti:Sa lasers will make a push to increase either the peak power or the repetition rate. Notably are the Centre for Advanced Laser Applications (CALA) facility in Munich which pushes to $3 \mathrm{PW}$ at several Hz, multi PW upgrades to the University of Michigan Hercules laser, and the CILEX ApolloN 10 PW laser at $>1$ shot/min, in France.

\section{High Energy Glass Laser Systems}

Several laser systems strive for more energetic pulses. The scaling of various acceleration concepts, especially ion acceleration, benefits from higher pulse energy to achieve either higher accelerated energies or more charge per bunch.

In short pulse laser systems the energy per pulse is a compromise between several constraints, such as damage fluence on optics, the accumulation of the nonlinear phase or B-integral, bandwidth requirements for short pulses, extraction efficiency, and size of the laser gain material. One of the most widely used laser material for high energy is Nd:glass, which can be produced in meter scale size with high optical and thermo-mechanical quality. It has been the material of choice for high energy fusion laser studies and was used to produce the first petawatt pulses. 
Nd:glass has a saturation fluence near damage fluence of ns pulses and is therefore suited of efficient storage and extraction of laser energy. The fluorescence bandwidth of the glass limits the pulse duration to $\sim 0.5 \mathrm{ps}$ for typical kJ lasers due to gain narrowing.

This limitation has been partially overcome by limiting the total gain in glass to about two orders of magnitude in the hybrid OPCPA Nd:glass Texas Petawatt laser. The laser uses a balanced mixture of two types of Nd:glass, silicate and phosphate, to keep the maximum bandwidth after the oscillator have been amplified to $1 \mathrm{~J}$ using OPCPA. The slow repetition rate of energetic laser has been overcome by a liquid cooled disk amplifier. This laser has the capability to operate at high energy and at moderate repetition rate.

\section{High Peak Power Laser Systems}

High peak power is often the primary parameter that is associated with a laser system, although it is certainly not the only critical parameter. Several petawatt lasers are now in operation and their pulse durations vary from tens of femtoseconds to sub-picosecond range. The high peak power lasers can be grouped by host material and technology. Figure 1 shows such a chart with several groups ranging from high energy glass lasers (typically not used for acceleration), petawatt glass lasers, hybrid systems, OPCPA systems to Ti:Sa lasers and to the ultra-broadband photon field synthesizer (PFS).

Although the peak power of lasers has been at $1 \mathrm{PW}$ for more than a decade now, it will very likely increase to $10 \mathrm{PW}$ or more in the next few years based on several projects that are funded by the Extreme Light Infrastructure in

Europe and similar proposals in other countries. $100 \mathrm{PW}$ pulses could be possible with OPCPA or hybrid OPCPA/glass systems, while exawatt concepts are based on beam combining of multiple lasers. Peak power itself is currently not considered the limiting factor for future accelerator driver, but the combination of peak power with average power and efficiency presents a challenge.

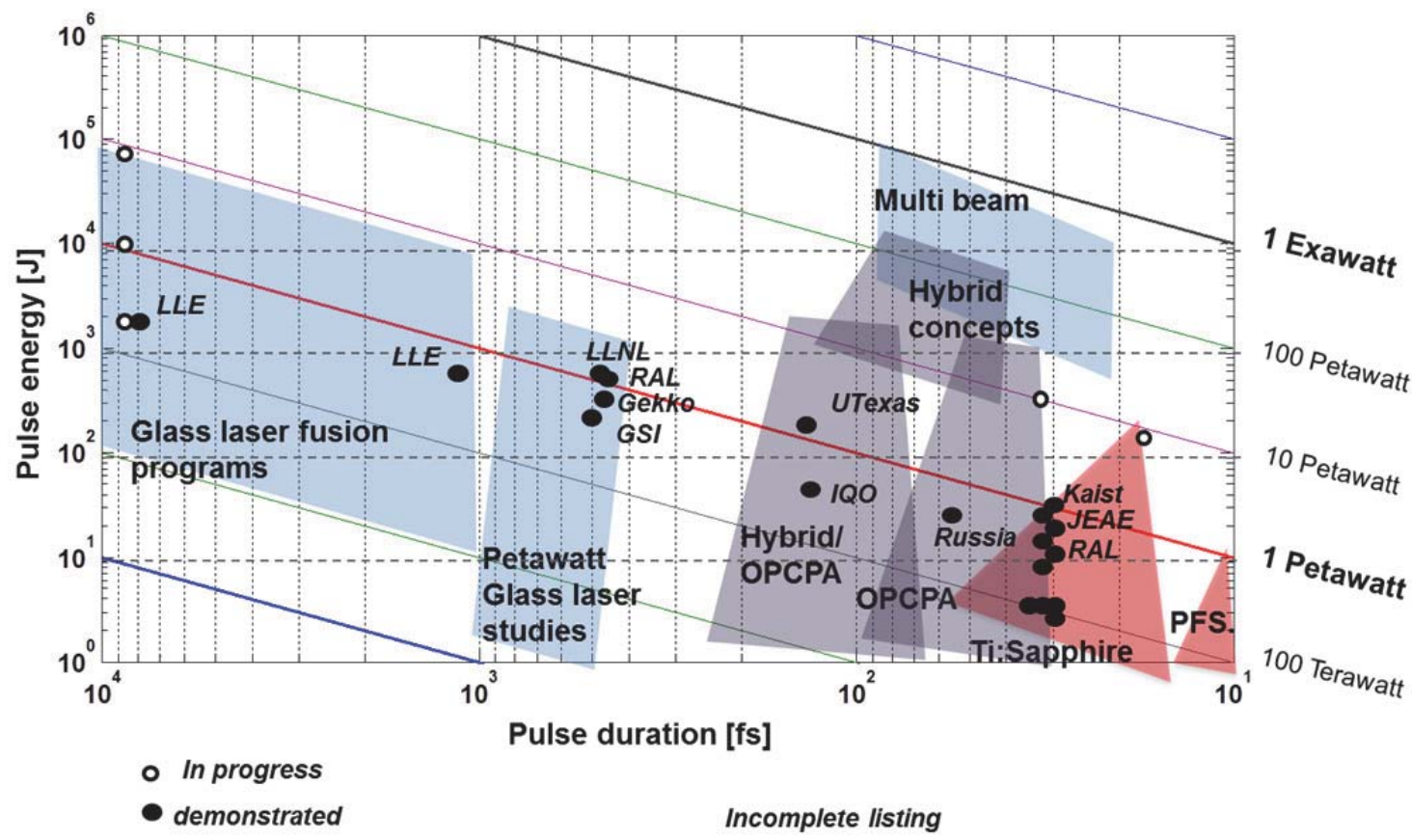

FIGURE 1. This chart groups the lasers technologies for high peak power systems. The colored areas show the possible extend of each technology. The dots indicate some examples of current systems.

\section{Alternate Wavelength Laser Systems}

The wavelength of the emitted laser radiation for most systems is determined by the laser host material, but it can also be driven by the particular application. Most laser use Neodymium or Ytterbium ions in a host crystal with a laser wavelength near $1060 \mathrm{~nm}$. The Ti:Sa crystal has a wide tuning range, but the emission peak is near $800 \mathrm{~nm}$. A 
clear exception is the $\mathrm{CO}_{2}$ laser with $\sim 10600 \mathrm{~nm}$ wavelength and was used for early plasma beat-wave experiments. All of these wavelengths have been chosen because the host material operates at its wavelengths and the solid state laser crystal is broadband enough to reach high peak power pulses. The scaling of LWFA or ion acceleration is not dominated by wavelength.

Dielectric accelerating structures are always designed to the RF frequency of the driving electric field. This is also the case for driving fields in the optical range. For current Dielectric Laser Accelerators (DLAs) the semiconductor etching technology is more suitable for few $\mu \mathrm{m}$ structures rather than $800 \mathrm{~nm}$ or $1060 \mathrm{~nm}$. OPCPA has the option of choosing the laser wavelength based on the phase matching and transparency range of optical crystals which far exceeds the lasing restriction of the host crystal. Suitable high peak power crystals can be found from 200-20000 nm and offer a wide selection for OPCPA. However, not all options lead to high peak power systems, and high damage threshold broadband gratings are not yet readily available for every wavelength. Besides OPCPA, there are several host materials that can push into the infrared range such as Thulium and Holmium doped host materials.

\section{Contrast, Timing, and Beam Quality}

Much more attention has been paid to the quality of laser pulses over the past years. Fiber lasers have very good beam quality, longterm reliability and stability. However, difficulties arise often with high energy pulses, which require larger aperture and more amplifier stages. The wavefront is typically corrected with a deformable mirror system consisting of a Shack-Hartmann or similar wavefront sensor and a mirror with an array of actuators to control the surface shape and therefore the wavefront of the reflected beam.

A high "pulse to pre-pulse" contrast is required for most solid target interaction. A laser focused to $10^{21} \mathrm{~W} / \mathrm{cm}^{2}$ will affect the target with a pre-pulse that is ten orders of magnitude lower than the peak. Amplified spontaneous emission (ASE) in amplifiers or parasitic fluorescence in OPCPA systems as well as scattering of optics contribute to the background of the pre-pulse level. Ghost reflections of multi-pass amplifiers or regenerative amplifiers cause short pre-pulses. Imperfect dispersion and phase control adds to the ramp before the main pulse. All of these phenomena affect the temporal shape of the pulse orders of magnitude below the main pulse and must be addressed. There are multiple approaches to reduce the pre-pulse level. Limiting the gain in every amplifier stage will reduce the ASE level and fluorescence level, but also reduce the efficiency. In double CPA the pulse is amplified in a CPA system, then cleaned and amplified again to higher peak power in a second CPA stage. The cleaner is for example cross polarization wave generation (XPW) or OPCPA, where the output pulse has a cubic dependency on the intensity of the input pulse and significantly reduces the pre-pulse level. These pulse cleaners eliminate pre-pulse that arise before the cleaner. Pulse cleaner at the end of the laser chain are for example a thin doubling crystals or plasma mirrors, which reflect only after the intensity is sufficiently high to turn the non reflecting surface into plasma and reflect the pulse. The drawback with this process the efficiency of only about $70 \%$, the difficulty of aligning a non reflecting surface and that plasma mirrors have to be replaced after almost every shot. Diagnostics to measure pre-pulses have been demonstrated with a high sensitivity range of 14 orders of magnitude.

\section{High Average Power and High Efficiency Laser Systems}

High average power is primarily driven by the requirement for high luminosity. This puts then a wall-plug-tolaser energy efficiency of tens of percent on the system to cut down the total operating and construction cost of a future multi- $\mathrm{TeV}$ class facility to an acceptable limit. High average power benefits would also apply to a smaller laser suitable for example for future RF sources or ion acceleration for medical purposes ( $200 \mathrm{MeV} /$ nucleon).

The overall efficiency in laser output is primarily determined by: 1. electrical power to optical power of the pump efficiency, 2. optical-to-optical coupling of pump light into the gain medium, 3. extraction of the gain medium, quantum defect, 4. optical losses in the amplifiers, compressor and optics, and 5. facility losses, power conditioning and optional cryogenic cooling. It is clear that flashlamp pumping is certainly too lossy. Ti:Sa, which is currently the workhorse CPA laser, uses a frequency doubled infrared laser as a pump source, has a large quantum defect and is a quasi-three level system, and will ultimately not reach high enough efficiency to be suitable for large scale laser plasma accelerators. 
Figure 2 shows a chart of a few typical systems that are currently (year 2012) available, where Hercules and BELLA represent Ti:Sa lasers, TexasPW and Omega-EP represent energetic glass lasers, and ELI (the European Extreme Light Infrastructure) represents a host of solid state lasers that will be built with significant funding in the next 2-4 years. It is clear that average power is improving, but none of them reaches at this time the required parameter space of future accelerators, therefore more research and funding will be needed to explore the most viable route toward efficient accelerator applications. Dielectric laser accelerators and medical ion sources are also among the goals that can be reached by further developing the current laser technology.

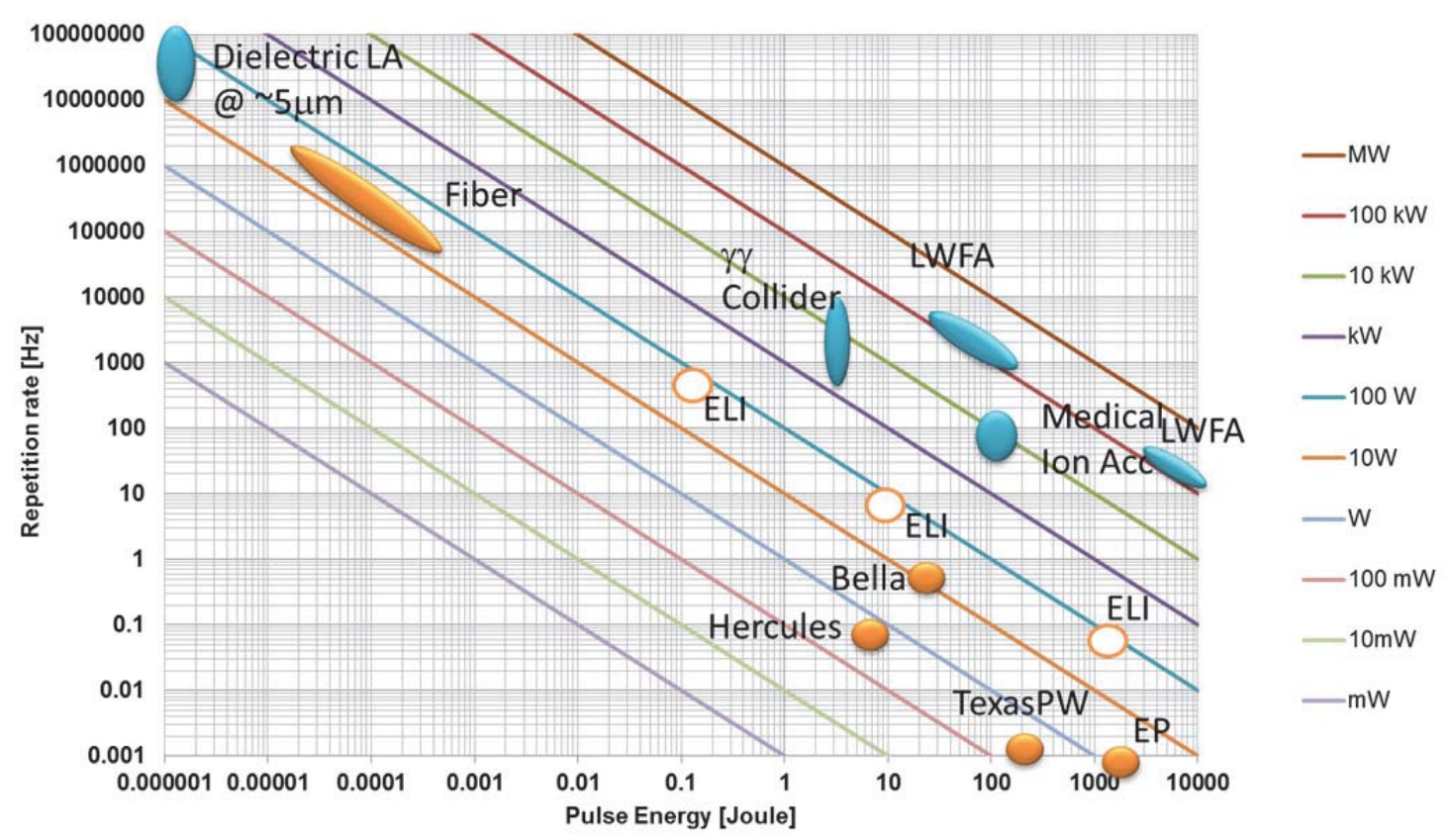

FIGURE 2. This chart shows in blue the wide range of energy and repetition rate requirements based on ref [1]. A few representative examples of Ti:sapphire lasers (BELLA, Hercules) and glass lasers or glass/hybrid (Omega EP, TexasPW) and the current status of pulsed fibers are indicated. The ELI circles represent current projects to be completed in a few $(\sim 4)$ years.

\section{SUMMARY}

Laser technology development for high peak and also high average power systems is a very active field. Since there is a wide variety of accelerator concepts and therefore a wide variety of matching lasers technologies, it is not straightforward to identify a single future laser technology to focus on and will certainly require further investigations. On the other hand, the lasers have made significant progress in peak power, average power, beam quality and reliability to advance the experimental progress. Laser technology and experimental progress goes hand in hand with narrowing down to future accelerators.

\section{ACKNOWLEDGMENTS}

We are grateful to the Department of Energy for their support of this work, and to all the Working Group 8 participants as well as the joint Working group participants for sharing their research results.

\section{REFERENCES}

1. ICFA Beam Dynamics Newsletters No 56, W. Leemans, W. Chou, M. Uesaka, 2011 